\title{
Maladies et plantes vasculaires du Karité (Vitellaria paradoxa Gaertn) au Bénin
}

\author{
Valerien A. Zinsou \\ André A. Fanou \\ Gildas C. Tchemadon \\ Huguette A. Dossa \\ Judith A. P. Doumatey \\ Balkissou Amadou Sambo Poulo \\ Rosalie Houénoumadin
}

Laboratoire de Phytotechnie, d'Amélioration et de Protection des Plantes,

Faculté d'Agronomie, Université de Parakou, Bénin

\section{Armand K. Natta}

Laboratoire d'Ecologie, de Botanique et de Biologie Végétale, Faculté

d'Agronomie, Université de Parakou, Bénin

Doi:10.19044/esj.2021.v17n10p270

Submitted: 28 October 2020

Accepted: 08 February 2021

Published: 31 March 2021
Copyright 2021 Author(s)

Under Creative Commons BY-NC-ND

4.0 OPEN ACCESS

Cite As:

Zinsou V.A., Fanou A.A., Tchemadon G.C., Dossa H.A., Doumatey J.A.P., Sambo Poulo B.A., Houénoumadin R. \& Natta A.K. (2021). Maladies et plantes vasculaires du Karité (Vitellaria paradoxa Gaertn) au Bénin. European Scientific Journal, ESJ, 17(10), 270. https://doi.org/10.19044/esj.2021.v17n10p270

\section{Résumé}

La présente étude vise à évaluer les maladies et les plantes vasculaires du karité (Vitellaria paradoxa C. F. Gaertn) dans les savanes Soudanienne (SS) et Nord guinéenne (SNG) du Bénin. Pour ce faire, quarante sites au total ont été prospectés au cours du mois de juillet 2018. Sur chaque site, l'incidence et la sévérité des symptômes de maladies, la densité et la distribution des plantes vasculaires ont été étudiées. Des échantillons de feuilles malades, des plantes vasculaires du karité ont été prélevés pour l'identification au laboratoire. Lasiodiplodia theobromae, Colletotrichum gloeosporioides, Pestalotiopsis sp, Xanthomonas sp ont été les agents pathogènes identifiés. Les valeurs d'incidence étaient élevées dans la SS (59,6\% L. theobromae, et de 42,2\% Xanthomonas sp) et dans la SNG (67,5\% Pestalotiopsis sp et de 55\% 
L. theobromae). Les valeurs de sévérité étaient élevées dans la SS $(39 \% \mathrm{~L}$. theobromae et de 30,6\% Xanthomonas sp) et dans la SNG (54,7\% Pestalotiopsis sp et $43 \%$ L. theobromae). Les données collectées sur la densité des plantes vasculaires ont montré une Orchidiaceae holo-épiphyte (Calyptrochilum christyanum), une Moraceae hémi-épiphyte (Ficus thonningii), une Renonculaceae (Alafia barteri) et deux Loranthaceae (Tapinanthus dodoneifolius, Tapinanthus globiferus). T. dodoneifolius et $T$. globiferus sont plus répandus avec des taux d'infestation moyens respectifs de $68,67 \%$ et de $66,58 \%$ et des densités moyennes par plant respectives de 5,64 touffes et de 2,52 touffes. Ainsi, les efforts d'amélioration de la production de karité doivent prendre en compte les stratégies de lutte contre ces agents pathogènes et plantes vasculaires.

Mots-clés: Karité, maladies, plantes parasites, plantes épiphytes, Vitellaria paradoxa

\title{
Diseases and vascular plants of shea-butter tree (Vitellaria paradoxa Gaertn) in Benin
}

\author{
Valerien A. Zinsou \\ André A. Fanou \\ Gildas C. Tchemadon \\ Huguette A. Dossa \\ Judith A. P. Doumatey \\ Balkissou Amadou Sambo Poulo \\ Rosalie Houénoumadin
}

Laboratoire de Phytotechnie, d'Amélioration et de Protection des Plantes,

Faculté d'Agronomie, Université de Parakou, Bénin

\section{Armand K. Natta}

Laboratoire d'Ecologie, de Botanique et de Biologie Végétale, Faculté

d'Agronomie, Université de Parakou, Bénin

Abstract

The present study aims to assess diseases and vascular plants of the shea-butter tree (Vitellaria paradoxa C. F. Gaertn) in Sudan Savanna (SS) and Northern Guinea Savanna (NGS) of Benin. Thus, forty (40) sites in total were surveyed in July 2018. On each site, incidence and severity of the disease symptoms, and density and distribution of vascular plants of the shea-butter tree were studied. Samples of diseased leaves and vascular plants of the sheabutter tree were collected for laboratory identification. The pathogens 
Lasiodiplodia theobromae, Colletotrichum gloeosporioides, Pestalotiopsis $\mathrm{sp)}$ and Xanthomonas sp were identified. The incidence values were high in SS (59.6\% L. theobromae and $42.2 \%$ Xanthomonas sp) and in NGS (67.5\% Pestalotiopsis sp and 55\% L. theobromae). The severity values were high in SS (39\% L. theobromae and 30.6\% Xanthomonas sp) and in NGS (54.7\% Pestalotiopsis sp and $43 \%$ L. theobromae). Data collected on the density of vascular plants of the shea-butter tree showed one holo-epiphytic Orchidiaceae (Calyptrochilum christyanum), one Moraceae (Ficus thonningii), one Renonculaceae (Alafia barteri) and two Loranthaceae (Tapinanthus dodoneifolius, Tapinanthus globiferus). T. dodoneifolius and T. globiferus were more widespread with respectively infestation rate of $68.67 \%$ and $66.58 \%$, and respectively average densities per shea plant of 5.64 tufts and 2.52 tufts. Thus, efforts to improve shea-butter production must take into account strategies for disease pathogens and vacular plants control in the sheabutter parklands in Benin.

Keywords: Shea-butter tree, diseases, parasitic plants, epiphytic plants, Vitellaria paradoxa

\section{Introduction}

Le karité (Vitellaria paradoxa) est un arbre de la famille des sapotacées qui pousse exclusivement en Afrique, sous le climat sahélosoudanien ou soudano guinéen et sur les sols argileux (Ahouansou et al., 2008). En Afrique, son aire de répartition s'étend sur une bande de $5.000 \mathrm{~km}$ de long sur 400 à $750 \mathrm{~km}$ de large du Sénégal oriental à l'Ouganda passant par le Burkina Faso, le Togo et le Nigéria et couvre 16 pays (Samaké et al., 2011). La sous-espèce nilotica est rencontrée entre 1200-1600 m d'altitude en Afrique centrale, en Afrique du Nord et en Afrique de l'Est, en grande quantité en Ouganda et Soudan. Quant à la sous-espèce paradoxa, elle est retrouvée à des altitudes comprises entre 100 et $600 \mathrm{~m}$, au Bénin, au Burkina Faso, au Cameroun, en République Centrafricaine, en Côte d'Ivoire, au Ghana, en Guinée, en Guinée Bissau, au Mali, au Niger, au Nigéria, au Sénégal, au Tchad et au Togo (Lafleur, 2008). Le karité (Vitellaria paradoxa) a été signalé au Bénin comme la deuxième sur la liste des 10 espèces ligneuses alimentaires prioritaires pour le Bénin (Eyog Matig et al., 2002). Il représente le troisième produit d'exportation du Bénin, à fort potentiel exportable avec une qualité fortement cotée sur les marchés régionaux et internationaux. Il joue un rôle économique très important dans les zones de production en termes de génération de revenus et de création d'emplois, et contribue à $1,23 \%$ au PIB (MAEP, 2008).

Le karité est très recherché aujourd'hui pour sa noix qui a une amande riche de 45 à $50 \%$ en matière grasse (Ahouansou et al., 2008). En effet, en 
saison des pluies, $V$. paradoxa subsp. paradoxa produit des baies semblables à de petits avocats à pulpe comestible. Le beurre extrait des amandes de l'espèce, appelé beurre de karité génère non seulement des revenus importants aux ménages de la région mais aussi entre dans la préparation de la nourriture des populations (Larrue, 2005). Ce beurre, est donc utilisé dans l'alimentation humaine, la cosmétologie et la pharmacologie (Ahouansou et al., 2008 ; Campbell, 2002). C'est surtout son intense utilisation alimentaire, notamment dans la chocolaterie, qui a donné un regain d'intérêt pour ce produit (Ahouansou et al., 2008). En effet, l'Union Européenne a autorisé l'incorporation des huiles végétales et notamment le beurre de karité, comme substitut au beurre de cacao dans les chocolats (Kapseu et al., 2001). La proportion de la substitution atteint même déjà $8 \%$ voire $15 \%$ car les huiles de substitution reviennent de 10 à $40 \%$ moins chères. Le karité est récolté par environ 4 millions de femmes dans la région Ouest Africaine et ses fruits sont d'une importante et croissante source de revenu pour les familles et communautés locales (Kpadonou et al., 2012 ; Gnanglè et al., 2012).

Au Bénin, le karité revêt également d'une importance socioculturelle, économique, médicinale, alimentaire, et agroforestière (Gnanglè et al., 2009; Sodjinou, 2006 ; Codjia et al., 2003). En effet, il constitue avec le néré, les espèces ligneuses les plus utilisées dans les parcs et systèmes agroforestiers traditionnels (Agbahungba et Depommier, 1989). De plus, avec une capacité de transformation de 12000 à 35000 tonnes de noix de karité récoltées pour l'exportation, le Bénin offre d'énormes potentialités sur le marché international (Akouehou et al., 2013).

Malgré cette si grande valeur qui semble lui être accordée de nos jours, le karité est menacé de disparition non seulement en raison du vieillissement des parcs, de sa faible régénération et la lenteur de sa croissance (Samaké et al., 2011), mais également à cause des systèmes de culture (Kindt et al., 2008). La dégradation des conditions climatiques et des sols, la pression humaine, mais aussi les phytopathogènes sans oublier les parasites du genre Tapinanthus, menacent également l'espèce et constituent des obstacles majeurs à la conservation des ressources génétiques des essences spontanées. En dépit de ces nombreuses études, la pathologie de l'espèce reste un domaine encore très peu explorée (Bayala et al., 2002; 2009) tout comme celui des parasites végétaux de l'espèce. Pour ce qui concerne les derniers (parasites du genre Tapinanthus), Boussim et al. (1993) ont rapporté qu'au Burkina Faso, $95 \%$ de la population de karité des savanes serait infestée par quatre espèces de Loranthaceae, à savoir : Agelanthus dodoneifolus (DC.) Polhill et Wiens, Tapinanthus bangwensis (Engl et K. Krause) Danser, Tapinanthus globiferus (A. Rich) Tiegh et Tapinanthus ophioides (Sprague) Danser. Au Bénin, des études sur la diversité des Loranthaceae et leur impact sur Vitellaria paradoxa C.F.Gaertn (Ahamidé et al., 2017) ont été effectués. Néanmoins la 
connaissance, des taux d'infestations et la densité parasitaire de chaque espèce de Loranthaceae et épiphytes, des maladies inféodés du Karité est nécessaire et peut contribuer à anticiper sur les moyens de lutte. C'est dans ce contexte que s'inscrit cette étude sur les maladies et plantes vasculaires du Karité au Bénin.

\section{Matériel et Méthodes}

\subsection{Milieu d'étude}

Les études ont été réalisées sur quarante (40) sites de production de karité distants de $5 \mathrm{~km}$ au moins, à raison de vingt-quatre (24) dans la savane Nord-guinéenne et seize (16) dans la savane Soudanienne au cours du mois de Juillet 2018 (Figure 1). La savane Soudanienne (SS) s'étend entre une latitude de 10.5 à $12^{\circ}$ et jouit d'une saison pluvieuse couvrant une période de Mai à Septembre avec une pluviométrie de $900-1000 \mathrm{~mm}$ et une température de 28 à $45^{\circ} \mathrm{C}$. Un sol ferrugineux tropical plus ou moins sablonneux caractérise cette savane. La savane Nord guinéenne est localisée entre une latitude 8.5 à $10.5^{\circ}$ et est marquée par une saison pluvieuse allant d'Avril à Septembre avec une pluviométrie comprise entre 1000 - $1100 \mathrm{~mm}$ et une température variant de 28 à $40^{\circ} \mathrm{C}$. Le sol est de type ferralitique rouge, avec des vertisols et des sols hydromorphes par endroit plus ou moins sableux ou argileux, (Azontondé, 1991).

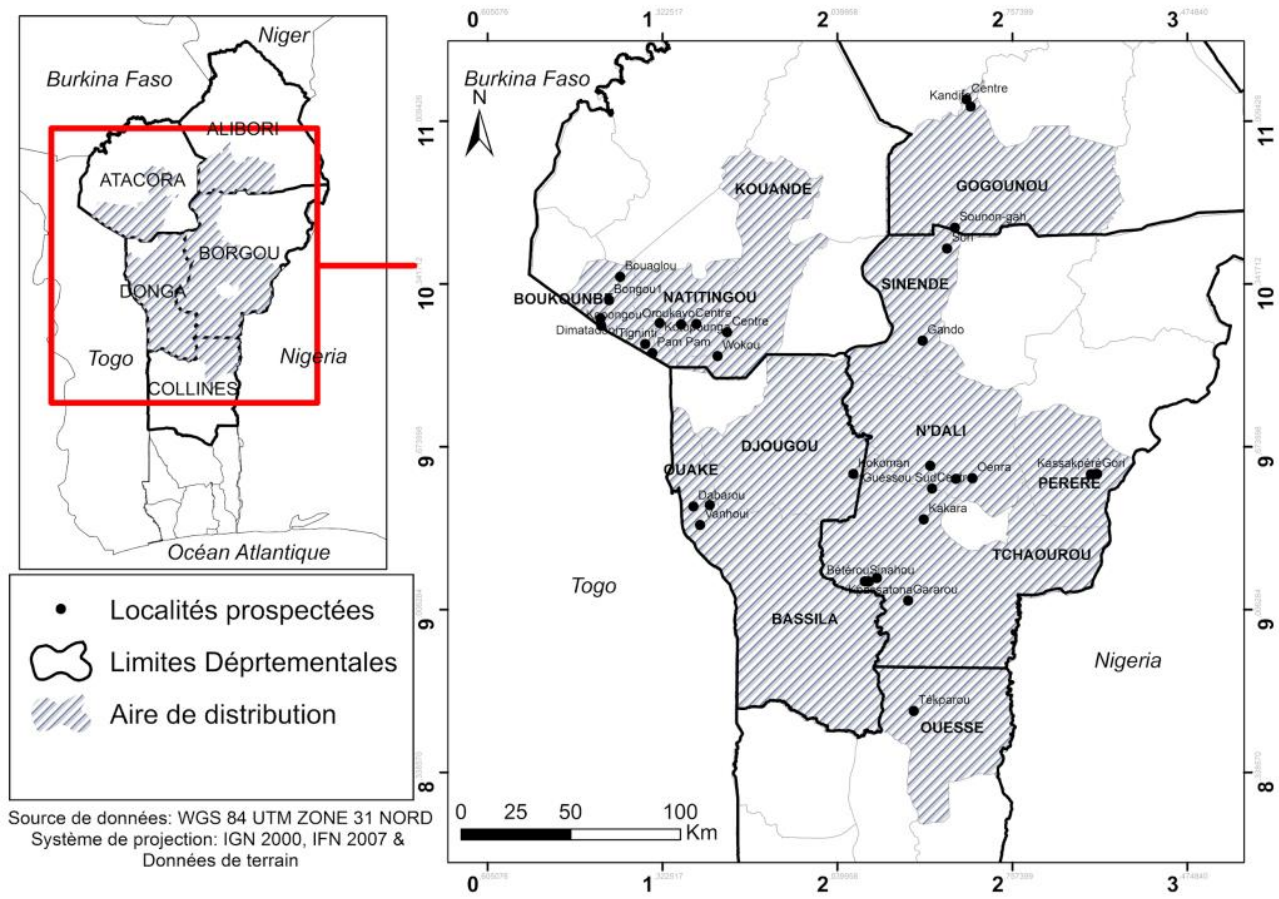

Figure 1 : Carte présentant les sites prospectés dans les savanes Nord guinéenne et Soudanienne du Bénin 


\subsection{Prospection}

La méthode de recensement de surface a été utilisée par la mise au point des placeaux d'un hectare. Au total, 40 placeaux d'un hectare ont été délimités. Dans chaque placeau, un échantillon de 10 pieds de $V$. paradoxa a été choisi et évalué suivant la méthode des diagonales. L'incidence et la sévérité ont évaluées pour chaque maladie. La sévérité de ces maladies a été évaluée sur les pieds à l'aide de l'échelle visuelle de 0 à 5 proposée par Sijaona et al. (2001) avec : $0=$ pas de symptôme ; $1=<1 \% ; 2=1-10 \%, 3=11-25 \%$ $; 4=26-50 \% ; 5=>50 \%$ ) de lésions par feuille infectée. De même, l'incidence des pieds parasités et la densité parasitaire des pieds de karité ont été évaluées par champ. Les espèces des plantes parasites et épiphytes ont été recensées par comptage du nombre de touffes pour chaque espèce de plantes parasites rencontrée sur chaque pied de karité, par placette et en fonction du diamètre à hauteur d'Homme (dbh), à 1,3 m du sol. Des échantillons de feuilles ont été prélevés sur la base des types de symptômes visibles de maladies et conservés dans des papiers journaux mis sous presse et transportés au Laboratoire de Phytotechnie, d'Amélioration et de Protection des Plantes (LaPAPP) de la Faculté d'Agronomie de l'Université de Parakou pour l'identification des agents pathogènes.

\subsection{Identification des agents pathogènes, plantes parasites et épiphytes}

Pour l'identification des champignons, les échantillons ont été prélevés de la partie incluant les tissus malades et sains, découpés puis stérilisés en surface avec une solution de $0,35 \%$ d'hypochlorite de sodium pendant une minute, rincés trois fois à l'eau stérile. Une petite portion de cette échantillon a été déposée dans une boite de Pétri contenant un milieu de culture Pomme de terre Dextrose Agar (39g/l) préalablement stérilisé et additionné de $5 \mathrm{ml}$ de solution d'acide citrique, avec trois répétitions pour chaque type d'isolat. Les boites ont été ensuite incubées à $25^{\circ} \mathrm{C}$ pendant $72 \mathrm{~h}$. Les cultures pures des champignons ont été réalisées par transfert de fragments mycéliens des isolats obtenus dans une boite de Pétri, sur milieu de culture PDA avec deux répétitions pour chaque isolat. Les boites ont été ensuite incubées à $25^{\circ} \mathrm{C}$ pendant 7 jours. Après fructification, l'identification de l'agent causal de la maladie a été réalisée sous microscope optique ZEISS, grâce à une clé sur la base des caractéristiques microscopiques ( nature du mycélium, morphologie et formation du conidiophore, arrangement des conidies sur les conidiophores, forme et taille des conidies, type et nombre de conidies, etc.) (Watanabe, 1994).

Pour l'identification des bactéries, des portions d'échantillons de feuille stérilisés en surface ont été rincés à l'eau stérile puis broyés dans $1 \mathrm{ml}$ de $0,01 \mathrm{M}$ solution de sulfate de magnésium préalablement stérilisé à 
l'autoclave. Le filtrat de chaque homogénat a été ensuite dilué six fois à 1/10 puis $100 \mu \mathrm{l}$ de la dernière dilution a été étalé dans deux boites de Pétri contenant de l'agar nutritif stérilisé à l'autoclave ( $23 \mathrm{~g} / \mathrm{l})$ additionné de solution de cycloheximide $(500 \mathrm{ppm})$. Les boites ont été ensuite incubées à $30^{\circ} \mathrm{C}$ pendant 48 à $72 \mathrm{~h}$. Les colonies isolées de Xanthomonas sp. ont été ensuite purifiées puis suspendues dans la solution de sulfate de magnésium pour obtenir une suspension aqueuse de cellules $\left(\mathrm{OD}_{660}=0,2\right.$ équivalent $10^{8}$ $\mathrm{CFU} / \mathrm{ml})$. Les suspensions ont été infiltrées à des feuilles de plants de karité, sous serre à l'aide d'une pompe, 4 semaines après semis dans des pots contenant un substrat (sol) stérilisé à $65^{\circ} \mathrm{C}$ pendant 72 heures, puis mélangé à du compost au rapport de $3 / 4$ (sol) et $1 / 4$ (compost). Les symptômes obtenus ont été comparés à ceux du champ.

L'identification des espèces de plantes parasites et épiphytes a été effectuée au Laboratoire d'Ecologie, de Botanique et de Biologie Végétale (LEB) de la Faculté d'Agronomie de l'Université de Parakou, à base des échantillons de rameaux recueillis sur le terrain et au moyen de la Flore Analytique du Bénin (Akoègninou et al., 2006) et arbres, arbustes et lianes des zones sèches d'Afrique de l'ouest (Arbonnier, 2002).

\subsection{Traitement et analyse des données}

La richesse spécifique en plantes parasites et épiphytes a été déterminée par pied de $V$. paradoxa. Pour tenir compte du fait que le nombre de touffe variait par parasite identifié, l'indice de diversité de ShannonWeaver H (Hill, 1973) a été calculé en utilisant la formule suivante : $\mathrm{H}=$ $\sum_{i=1}^{S} \mathrm{Pi} \log 2 \mathrm{Pi} \sum_{i=1}^{S} \mathrm{Pi} \log 2 \mathrm{Pi}$; avec $\mathrm{S}=$ la richesse spécifique, Pi la proportion du nombre d'individu de l'espèce i. L'incidence des champs parasités a été calculée. L'évaluation de la densité parasitaire par pied de karité a permis de classifier la vulnérabilité des plants suivant les cinq niveaux d'infestation proposés par Houéhanou et al. (2011) : N1: très faible infestation (1-5 touffes sur un pied), N2: faible infestation (6-10 touffes sur un pied), N3: infestation moyenne (11-15 touffes sur un pied), N4: infestation forte (16-20 touffes sur un pied), N5: infestation très forte (plus de 20 touffes sur un pied). L'analyse de variance (ANOVA) a été réalisée grâce au logiciel $R$ version 3.1.2 sur les valeurs d'incidence et densité parasitaire par pied des champs parasités, incidence et sévérité des maladies afin de comparer les moyennes au seuil de 5\%. Le test de Tukey a été complété afin de séparer les moyennes lorsqu'il y avait une différence significative $(\mathrm{p} \leq 0,05)$. Pour tester l'effet du diamètre à hauteur d'Homme (dbh) de l'arbre et de l'altitude sur le parasitisme et les maladies du karité, les régressions linéaires généralisées à effets mixtes ont été utilisées (Bolker et al., 2009) avec le package glm TMB (Brooks et al., 2017) dans $\mathrm{R}$ version 3.5.0 ( $\mathrm{R}$ Core Team 2018). La régression logistique 
binaire a été utilisée pour tester la présence ou non des plantes vasculaires et des maladies sur les arbres du karité.

\section{Résultats}

\subsection{Identification des maladies inféodées au $V$. paradoxa}

Quatre agents pathogènes ont été recensés comme responsables des pathologies notées sur le $V$. paradoxa dans les savanes Nord-guinéenne et Soudanienne du Bénin. Il s'agit des champignons (Lasiodiplodia theobromae, Pestalotiopsis sp et Colletotrichum gloesporioides) et de la bactérie Xanthomonas sp (figures 2, 3, 4, 5).

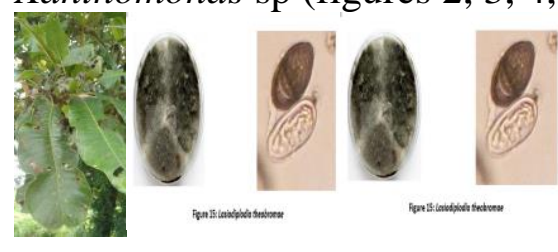

Figure 2 : (a) symptômes sur feuilles (b) L. theobromae sur PDA et (c) conidies au microscope (1000X)
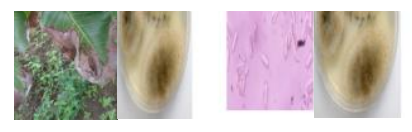

Figure 4 : (a) nécroses sur feuilles (b) $C$. gloeosporioides sur PDA et (c) conidies au microscope (400X)

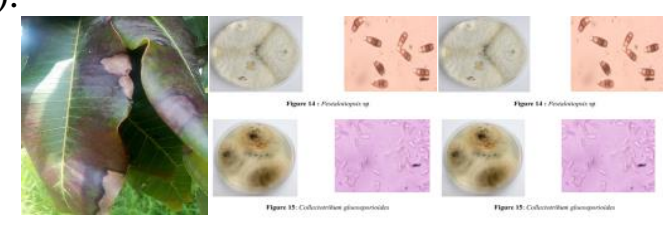

Figure 3 : (a) taches foliaires nécrotiques, b) Pestalotiopsis sp sur PDA et (c) conidies au microscope (400X)

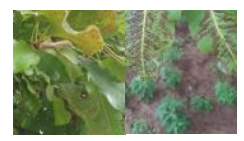

Figure 5 : Taches anguleuses nécrotiques sur feuilles par Xanthomonas sp.

\subsection{Distribution des maladies du karité}

Une différence significative a été observée entre les deux zones phytogéographiques tant pour l'incidence de Xanthomonas sp. $\left(\mathrm{p}<2 \mathrm{e}^{-16}\right)$, Pestalotiopsis sp. $\left(\mathrm{p}<2 \mathrm{e}^{-16}\right)$ et $C$. gloesporioides $(\mathrm{P}=0,034)$, que pour la sévérité de Xanthomonas sp. $\left(\mathrm{p}<2 \mathrm{e}^{-16}\right)$, Pestalotiopsis $\mathrm{sp} .\left(\mathrm{p}<2 \mathrm{e}^{-16}\right)$ et $C$. gloesporioides $(\mathrm{p}=0,049)$ (Tableau 1). Par contre aucune différence significative n'a été notée entre les deux zones phytogéographiques pour l'incidence et la sévérité de $L$. theobromae. Les fortes valeurs d'incidence $(67,54 \%)$ ont été enregistrées avec Pestalotiopsis sp. dans la savane Nord guinéenne ( $\mathrm{SNG}$ ) suivies de celles de $L$. theobromae respectivement de $59,69 \%$ et $55,07 \%$ dans la savane Soudanienne (SS) et la SNG, et de Xanthomonas sp. 42,27\% dans la SS. Les plus fortes valeurs de sévérité (54,70 $\pm 1,58)$ ont été observées avec Pestalotiopsis sp. dans la SNG suivies de celles de L. theobromae $(43,04 \%)$ dans la SNG, et de Xanthomonas sp. (30,68\%) dans la SS. C. gloesporioides n'a été retrouvé sur le karité que dans la savane 
Soudanienne à des valeurs d'incidence et de sévérité respectives de $0,65 \%$ et de $0,26 \%$.

Tableau 1: Incidence et sévérité des maladies inféodées au karité dans deux zones phytogéographiques

\begin{tabular}{|c|c|c|c|c|}
\hline \multicolumn{2}{|c|}{ Paramètre } & \multicolumn{2}{|l|}{ Savane Soudanienne } & \multirow{2}{*}{$\begin{array}{l}\text { Savane Nord } \\
\text { Guinéenne } \\
55,07 \pm 1,9 \mathrm{a}\end{array}$} \\
\hline L. theobromae & Incidence $(\%)$ & $59,69 \pm 2,29 a$ & & \\
\hline & $\mathrm{p}$ & & 0,121 & \\
\hline & Sévérité (\%) & $39,00 \pm 1,62 \mathrm{a}$ & & $43,04 \pm 1,59 b$ \\
\hline & $\mathrm{p}$ & & 0,086 & \\
\hline \multirow[t]{4}{*}{ Pestalotiopsis sp. } & Incidence $(\%)$ & $39,43 \pm 2,28 \mathrm{a}$ & & $67,54 \pm 1,78 b$ \\
\hline & $\mathrm{p}$ & & $<2 \mathrm{e}-16$ & \\
\hline & Sévérité (\%) & $28,80 \pm 1,79 a$ & & $54,70 \pm 1,58 b$ \\
\hline & $\mathrm{p}$ & & $<2 \mathrm{e}-16$ & \\
\hline \multirow[t]{4}{*}{ Xanthomonas sp. } & Incidence $(\%)$ & $42,27 \pm 2,31 \mathrm{a}$ & & $2,61 \pm 0,61 b$ \\
\hline & $\mathrm{p}$ & & $<2 \mathrm{e}-16$ & \\
\hline & Sévérité (\%) & $30,68 \pm 1,78 \mathrm{a}$ & & $1,91 \pm 0,46 b$ \\
\hline & $\mathrm{p}$ & & $<2 \mathrm{e}-16$ & \\
\hline \multirow[t]{4}{*}{ C. gloesporioides } & Incidence $(\%)$ & $0,65 \pm 0,38 \mathrm{a}$ & & $0 \pm 0 \mathrm{~b}$ \\
\hline & $\mathrm{p}$ & & 0,034 & \\
\hline & Sévérité (\%) & $0,26 \pm 0,16 a$ & & $0 \pm 0 b$ \\
\hline & $\mathrm{p}$ & & 0,0049 & \\
\hline
\end{tabular}

\subsection{Effet du diamètre des pieds de karité et de l'altitude sur l'incidence et la sévérité des maladies du karité}

Le Dbh a un effet significatif et positif, tant sur l'incidence $(\mathrm{p}=0,043$; $\mathrm{E}=0,151)$ que sur la sévérité $(\mathrm{p}=0,0268 ; \mathrm{E}=0,1251)$ de Xanthomonas $\mathrm{sp}$. (Tableau 2). Le Dbh a eu un effet négatif, hautement significatif $(\mathrm{p}=0,0085$; $\mathrm{E}=-0,2487)$ et significatif ( $\mathrm{p}=0,0318 ; \mathrm{E}=-0,1769)$ sur l'incidence et la sévérité de Pestalotiopsis sp. Il apparait donc que Xanthomonas sp. infecte et sévit plus sur les gros arbres contrairement à Pestalotiopsis sp. qui infecte et sévit plus sur les petits arbres.

L'altitude a un effet positif, significatif ( $\mathrm{p}=0,0396 ; \mathrm{E}=0,0376)$ et hautement significatif $(\mathrm{p}=0,0016 ; \mathrm{E}=0,0454)$, respectivement sur l'incidence et la sévérité de $L$. theobromae. L'altitude a un effet négatif, très hautement significatif sur l'incidence $\left(\mathrm{p}<2 \mathrm{e}^{-16} ; \mathrm{E}=-0,1648\right)$ et la sévérité ( $\mathrm{p}$ $<2 \mathrm{e}^{-16} ; \mathrm{E}=-0,1253$ ) de Xanthomonas sp. Au niveau de Pestalotiopsis sp., l'altitude a un effet positif, très hautement significatif, tant sur l'incidence ( $\mathrm{p}$ 
$\left.<2 \mathrm{e}^{-16} ; \mathrm{E}=0,2207\right)$ que sur la sévérité $\left(\mathrm{p}<2 \mathrm{e}^{-16} ; \mathrm{E}=0,1578\right)$. Il ressort que L. theobromae, Xanthomonas sp. et Pestalotiopsis sp. infectent et sévissent plus sur les plants de karité de hautes altitudes que ceux de basses altitudes.

Tableau 2: Diamètre des pieds de karité et de l'altitude en fonction de l'incidence et la sévérité des maladies du karité

\begin{tabular}{cccccc}
\hline & & $\begin{array}{c}\text { Dbh - } \\
\text { Incidence }\end{array}$ & $\begin{array}{c}\text { Dbh - } \\
\text { Sévérité }\end{array}$ & $\begin{array}{c}\text { Altitude - } \\
\text { Incidence }\end{array}$ & $\begin{array}{c}\text { Altitude - } \\
\text { Sévérité }\end{array}$ \\
\hline Lasiodiplodia & $\mathrm{E}$ & 0,112 & 0,074 & 0,038 & 0,045 \\
theobromae & $\mathrm{t}$ & 1,098 & 0,921 & 2,060 & 3,158 \\
& $\mathrm{p}$ & 0,273 & 0,357 & 0,040 & 0,002 \\
\hline Colletotrichum & $\mathrm{E}$ & 0,001 & 0,001 & $4,04.10^{-4}$ & $1,97.10^{-4}$ \\
gloesporioides & $\mathrm{t}$ & 0,069 & 0,330 & 0,214 & 0,242 \\
& $\mathrm{p}$ & 0,945 & 0,742 & 0,831 & 0,809 \\
\hline Pestalotiopsis $\mathrm{sp}$. & $\mathrm{E}$ & $-0,249$ & $-0,177$ & 0,221 & 0,158 \\
& $\mathrm{t}$ & $-2,634$ & $-2,149$ & 13,029 & 10,685 \\
& $\mathrm{p}$ & 0,009 & 0,032 & $<2 \mathrm{e}^{-16}$ & $<2 \mathrm{e}^{-16}$ \\
\hline & $\mathrm{E}$ & 0,151 & 0,125 & $-0,165$ & $-0,125$ \\
Xanthomonas $\mathbf{s p}$. & $\mathrm{t}$ & 2,026 & 2,218 & $-12,323$ & $-12,373$ \\
& $\mathrm{p}$ & 0,043 & 0,027 & $<2 \mathrm{e}^{-16}$ & $<2 \mathrm{e}^{-16}$ \\
\cline { 2 - 5 }
\end{tabular}

$\mathrm{Dbh}=$ diamètre à hauteur d'Homme; $\mathrm{E}=$ Estimate $; \mathrm{t}=\mathrm{t}$ value ;

$\mathrm{p}=$ probabilité; les moyennes suivies des mêmes lettres sur chaque ligne, ne sont pas significativement différentes au seuil de $5 \%$ pour le test de Turkey

\subsection{Diversité des plantes parasites et épiphytes du karité}

Cinq (05) espèces de plantes parasites et épiphytes ont été répertoriées (figure 6). Il s'agit de deux Loranthaceae (Tapinanthus dodoneifolius, Tapinanthus globiferus), d'une Orchidiaceae holo-épiphyte (Calyptrochilum christyanum), d'une Moraceae hémi-épiphyte (Ficus thonningii), et d'une Renonculaceae (Alafia barteri). T. dodoneifolius, $T$. globiferus et $C$. christyanum ont été relevés dans les deux savanes alors que Ficus thonningii et Alafia barteri ont été recensés dans la savane Soudanienne. La richesse spécifique (RS) a varié de 1 à 5 dans la savane Soudanienne et de 1 à 3 dans celle Nord guinéenne. Il existe une différence très hautement significative $(\mathrm{P}$ $<2 \mathrm{e}^{-16}$ ) de l'indice de Shannon $(\mathrm{H})$ entre les deux zones phytogéographiques étudiés. La diversité a été plus élevée dans la savane Soudanienne $(0,56 \pm$ $0,02)$ que dans celle Nord guinéenne $(0,35 \pm 0,02)$.
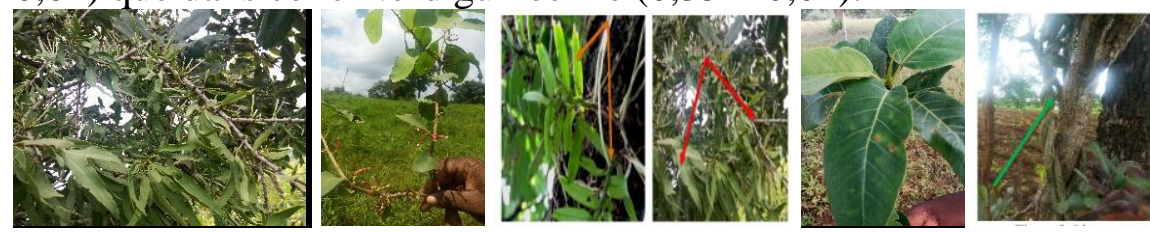

Figure 6: (a) T. dodoneifolius, (b) T. globuferus, (c) C. christyanum, (d) Ficus thonningii,

(e) Alafia barteri 


\subsection{Incidence, densité parasitaire par pied et niveau d'infestation des parasites et épiphytes}

Il existe une différence très hautement significative entre les deux zones phytogéographiques, en ce qui concerne l'incidence de Tapinanthus dodoneifolius $\left(\mathrm{P}=4,45 \mathrm{e}^{-12}\right)$ et Tapinanthus globiferus $\left(\mathrm{P}=2,03 \mathrm{e}^{-15}\right)$, contrairement aux autres espèces (Tableau 3 ). En effet, les plus fortes valeurs d'incidences ont été observées dans la savane Soudanienne $(80,17 \%$ et $79,96 \%)$ respectivement pour $T$. dodoneifolius et $T$. globiferus contre $(61,01 \%$ et $57,68 \%$ ) dans la savane Nord guinéenne. De même, une différence très hautement significative entre les deux zones a été notée par rapport à la densité parasitaire de $T$. dodoneifolius $\left(\mathrm{P}<2 \mathrm{e}^{-16}\right)$ et $T$. globiferus $\left(\mathrm{P}<2 \mathrm{e}^{-16}\right)$, contrairement aux autres espèces. En effet, les plus fortes valeurs de densité parasitaire ont été observées dans la savane Soudanienne ( 7,88 touffes et 3,75 touffes) respectivement pour $T$. dodoneifolius et $T$. globiferus contre 4,16 touffes et 1,71 touffes respectivement dans la savane Nord guinéenne.

Tableau 3: Variation de l'incidence et de la densité parasitaire moyenne par pied de karité

\begin{tabular}{|c|c|c|c|c|}
\hline Paramètre & & $\begin{array}{c}\text { Savane } \\
\text { Soudanienne }\end{array}$ & & $\begin{array}{c}\text { Savane Nord } \\
\text { Guinéenne }\end{array}$ \\
\hline Tapinanthus & Incidence $(\%)$ & $80,17 \pm 1,86 a$ & & $61,01 \pm 1,86 b$ \\
\hline \multirow[t]{3}{*}{ dodoneifolius } & & & $4,45 \mathrm{e}^{-12}$ & \\
\hline & $\begin{array}{c}\text { Densité parasitaire } \\
\text { (touffes) }\end{array}$ & $7,88 \pm 0,40 a$ & & $4,16 \pm 0,22 b$ \\
\hline & $\mathrm{p}$ & & $<2 \mathrm{e}^{-16}$ & \\
\hline \multirow{4}{*}{$\begin{array}{l}\text { Tapinanthus } \\
\text { globiferus }\end{array}$} & Incidence $(\%)$ & $79,96 \pm 1,87 \mathrm{a}$ & & $57,68 \pm 1,88 b$ \\
\hline & $\mathrm{p}$ & & $2,03 \mathrm{e}^{-15}$ & \\
\hline & $\begin{array}{c}\text { Densité parasitaire } \\
\text { (touffes) }\end{array}$ & $3,75 \pm 0,19 a$ & & $1,71 \pm 0,09 b$ \\
\hline & $\mathrm{p}$ & & $<2 \mathrm{e}^{-16}$ & \\
\hline \multirow{4}{*}{$\begin{array}{l}\text { Calyptrochilum } \\
\text { christyanum }\end{array}$} & Incidence $(\%)$ & $0,22 \pm 0,21 a$ & & $0 \pm 0 \mathrm{a}$ \\
\hline & $\mathrm{p}$ & & 0,22 & \\
\hline & $\begin{array}{c}\text { Densité parasitaire } \\
\text { (touffes) }\end{array}$ & $0,002 \pm 0,002 \mathrm{a}$ & & $0 \pm 0 \mathrm{a}$ \\
\hline & $\mathrm{p}$ & & 0,22 & \\
\hline \multirow[t]{4}{*}{ Ficus thonningii } & Incidence $(\%)$ & $0,22 \pm 0,22 \mathrm{a}$ & & $2,90 \pm 1,39 a$ \\
\hline & $\mathrm{p}$ & & 0,117 & \\
\hline & $\begin{array}{c}\text { Densité parasitaire } \\
\text { (touffes) }\end{array}$ & $0,01 \pm 0,006 \mathrm{a}$ & & $0,02 \pm 0,009 a$ \\
\hline & $\mathrm{p}$ & & 0,122 & \\
\hline \multirow[t]{4}{*}{ Alafia barteri } & Incidence $(\%)$ & $0,22 \pm 0,22 a$ & & $0 \pm 0 \mathrm{a}$ \\
\hline & $\mathrm{p}$ & & 0,22 & \\
\hline & $\begin{array}{c}\text { Densité parasitaire } \\
\text { (touffes) }\end{array}$ & $0,004 \pm 0,004 a$ & & $0 \pm 0 \mathrm{a}$ \\
\hline & $\mathrm{p}$ & & 0,22 & \\
\hline
\end{tabular}

$\mathrm{p}$ = probabilité; les moyennes suivies des mêmes lettres sur chaque ligne, ne sont pas significativement différentes au seuil de $5 \%$ pour le test de Turkey 
Les figures ( $7 \mathrm{a}$ et $7 \mathrm{~b})$ présentent les incidences par niveau d'infestation par zone phytogéographique pour chacune des espèces. Il ressort que l'incidence du faible niveau d'infestation (N1) est plus élevée dans la savane Nord guinéenne que dans la savane Soudanienne pour les Tapinanthus. En ce qui concerne l'incidence des autres niveaux d'infestation (N2 à N5), on note une proportion plus élevée dans la savane Soudanienne que dans celle Nord guinéenne pour les Tapinanthus.
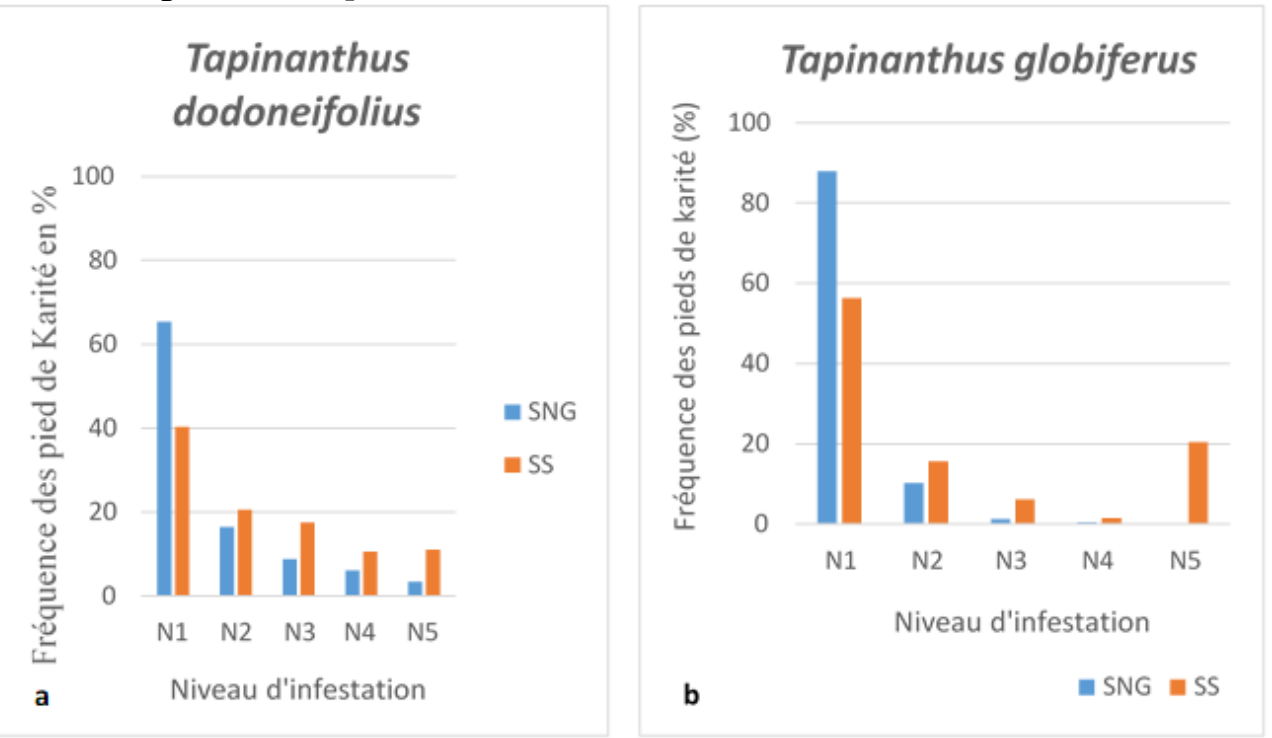

Figure 7 : Variation des pieds de karité en fonction du niveau d'infestation par zone phytogéographique de (a) T. dodoneifolius (b) T. globiferus

\subsection{Effet du diamètre des pieds de karité et de l'altitude sur l'incidence et la densité parasitaire}

Le Dbh a un effet très hautement significatif et positif, respectivement sur l'incidence et la densité parasitaire de T. dodoneifolius $\left(\mathrm{p}<2 \mathrm{e}^{-16} ; \mathrm{E}=0,979\right.$ et $\left.\mathrm{p}<2 \mathrm{e}^{-16} ; \mathrm{E}=0,224\right)$ et de $T$. globiferus $\left(\mathrm{p}=3,56 \mathrm{e}^{-15} ; \mathrm{E}=0,752\right.$ et $\mathrm{p}=1,9$. $10^{-6} ; \mathrm{E}=-0,033$ ) (Tableau 4). Un effet hautement significatif et positif a été noté sur l'incidence $(\mathrm{p}=0,005 ; \mathrm{E}=0,017)$ et la densité parasitaire $(\mathrm{p}=0,005$ $; \mathrm{E}=0,0002$ ) de $C$. christyanum. Il ressort donc que les gros arbres de karité sont plus infestés et peuplés par $T$. dodoneifolius, $T$. globiferus et $C$. christyanum, que les plus petits.

Quant à l'altitude, il a un effet très hautement significatif et négatif, respectivement sur l'incidence $\left(\mathrm{p}=1,74 \mathrm{e}^{-12} ; \mathrm{E}=-0,112\right)$ et la densité parasitaire $\left(\mathrm{p}=8,96.10^{-9} ; \mathrm{E}=-0,013\right)$ de $T$. dodoneifolius. Sur la densité parasitaire de T. globiferus, il a été aussi relevé, un effet significatif et négatif de l'altitude $(\mathrm{p}=0,0145 ; \mathrm{E}=-0,003)$. Ainsi, les plants de karité de hautes 
altitudes, sont moins infestés par $T$. dodoneifolius et moins peuplés par les deux Tapinanthus.

Tableau 4: Diamètre des pieds de karité et de l'altitude en fonction de l'incidence et de la densité parasitaire des plantes

\begin{tabular}{cccccc}
\hline & & $\begin{array}{c}\text { Dbh - } \\
\text { Incidence }\end{array}$ & $\begin{array}{c}\text { Dbh - Densité } \\
\text { parasitaire }\end{array}$ & $\begin{array}{c}\text { Altitude - } \\
\text { Incidence }\end{array}$ & $\begin{array}{c}\text { Altitude - } \\
\text { Densité } \\
\text { parasitaire }\end{array}$ \\
\hline Tapinanthus & $\mathrm{E}$ & 0,979 & 0,224 & $-0,112$ & $-0,013$ \\
dodoneifolius & $\mathrm{t}$ & 11,154 & 17,319 & $-7,132$ & $-5,792$ \\
& $\mathrm{p}$ & $<2 \mathrm{e}^{-16}$ & $<2 \mathrm{e}^{-16}$ & $1,74 \mathrm{e}^{-12}$ & $8,96.10^{-9}$ \\
\hline \multirow{2}{*}{ Tapinanthus } & $\mathrm{E}$ & 0,752 & 0,033 & $-0,026$ & $-0,003$ \\
globiferus & $\mathrm{t}$ & 7,979 & 4,789 & $-1,536$ & $-2,447$ \\
& $\mathrm{p}$ & $3,56 \mathrm{e}^{-15}$ & $1,9.10^{-6}$ & 0,125 & 0,015 \\
\hline \multirow{2}{*}{ Calyptrochilum } & $\mathrm{E}$ & 0,017 & $2.10^{-4}$ & $-0,001$ & $-1,34.10^{-5}$ \\
christyanum & $\mathrm{t}$ & 2,795 & 2,795 & $-1,233$ & $-1,233$ \\
& $\mathrm{p}$ & 0,005 & 0,005 & 0,218 & 0,218 \\
\hline \multirow{2}{*}{ Ficus thonningii } & $\mathrm{E}$ & 0,034 & $6,7.10^{-6}$ & $-0,012$ & $-2,15.10^{-5}$ \\
& $\mathrm{t}$ & 0,588 & 0,015 & $-1,127$ & $-0,277$ \\
& $\mathrm{p}$ & 0,557 & 0,988 & 0,260 & 0,782 \\
\hline \multirow{2}{*}{ Alafia barteri } & $\mathrm{E}$ & 0,008 & $1,66.10^{-4}$ & $4.10^{-4}$ & $7,95.10^{-6}$ \\
& $\mathrm{t}$ & 1,370 & 1,370 & 0,365 & 0,365 \\
\cline { 2 - 6 } & $\mathrm{p}$ & 0,171 & 0,171 & 0,715 & 0,715 \\
\hline
\end{tabular}

$\mathrm{Dbh}=$ diamètre à hauteur d'Homme; $\mathrm{E}=$ Estimate; $\mathrm{t}=\mathrm{t}$ value;

$\mathrm{p}$ = probabilité; les moyennes suivies des mêmes lettres sur chaque ligne, ne sont pas significativement différentes au seuil de $5 \%$ pour le test de Turkey

\section{Discussion}

Pestalotiopsis sp., Colletotrichum gloesporioides, Lasiodiplodia theobromae et Xanthomonas sp. ont été recensés comme responsables des pathologies notées sur le $V$. paradoxa dans les savanes Nord-guinéenne et Soudanienne du Bénin. Ces résultats corroborent ceux de Sakalidis et al. (2011), qui ont relevé Pestalotiopsis sp., Colletotrichum gloesporioides et Lasiodiplodia theobromae comme les trois principaux agents pathogènes du karité. De plus, Esiegbuya et al. (2014) ont aussi remarqué Pestalotiopsis sp. et Lasiodiplodia $s p$. sur des pieds de karité au Nigeria. Il convient également de noter que diverses études sur les fruitiers tels que le manguier (Dianda et al., 2018 ; Tédihou et al., 2017 ; Rehman et al., 2015), les citrus (Tédihou et al., 2017), le karité, et même le Daniellia oliveri (Akrofi et Amoah, 2009) ont signalé Lasiodiplodia theobromae, Pestalotiopsis sp.et Xanthomonas sp comme des menaces pour la production.

Les valeurs d'incidence de Lasiodiplodia theomobrae révélées par cette étude dans la savane Soudanienne $(59,69 \%)$ et celle Nord-guinéenne $(55,07 \%)$ sont inférieures à celles signalées par Tédihou et al. (2017) sur le manguier et les citrus de la région centrale du Togo, où ce champignon est le 
plus fréquent et présent dans $83,33 \%$ des tissus étudiés. Cependant, ces valeurs sont légèrement supérieures à celles retrouvées par Rehman et al. (2015) sur les racines et tiges du manguier au Pakistan $(43,11$ et $51 \%)$ ou par Dianda et al. (2018), sur le manguier au Burkina Faso (35,5\%).

La bactériose du karité causée par Xanthomonas sp. est à la base des taches translucides sur les feuilles du V. paradoxa. Ces mêmes symptômes ont été également enregistrés sur le manguier au Bénin par Zinsou et al. (2017) ; au Ghana par Pruvost et al. (2011) et signalé par Delhove et al. (2013).

L'étude a inventorié cinq espèces de plantes vasculaires sur le karité dans les savanes Soudanienne et Nord guinéenne du Bénin. Parmi elles, deux espèces prédominent. Il s'agit de Tapinanthus dodoneifolius et Tapinanthus globiferus. Ces deux espèces de la famille des Loranthaceae, ont été également signalées au Burkina Faso (Boussim et al., 1993), au Mali (Boussim, 2002), en Côte d'Ivoire (Amon et al., 2010), au Cameroun (Azo'o et al., 2013), au Nigéria (Odebiyi et al., 2004) et au Bénin (Akoègninou et al., 2006; Houénon et al., 2012; Ahamidé et al. 2017). Selon Akoègninou et al. (2006), au Benin, la diversité floristique en Loranthaceae est de sept espèces dans la même zone. Cependant notre étude n'a relevé que deux espèces de Loranthaceae. Cette différence peut s'expliquer par la méthode d'échantillonnage et les conditions climatiques. En effet, selon Ahamidé et al. (2017), les conditions climatiques influencent la répartition des Loranthaceae. Jiofack et al. (2007) ont signalé que les Loranthaceae se caractérisent par leur expansion importante d'une localité à l'autre en fonction de la variabilité des conditions écologiques. Il faut noter qu'il existe une forte affinité entre le Tapinanthus dodoneifolius et Tapinanthus globiferus comme l'ont déjà signalé, Ahamidé et al. (2017) sur le karité au Bénin et Boussim et al. (1993) au Burkina Faso. Ceci n'est pas le cas au niveau des agrumes et du colatier. En effet, les agrumes présentent une plus forte affinité avec T. globiferus (Houénon, 2012) tandis que le colatier (Cola nitida) a plutôt une forte affinité avec Phragmanthera capitata et Globimetula cupulata (Ahamidé et al., 2015).

Le taux moyen d'infestation des Loranthaceae dans la savane Soudanienne est de $80,07 \%$. Ce taux correspond à celui mentionné dans les parcs agroforestiers environnant le parc national de la Pendjari au Nord du Bénin par Houéhanou et al. (2011). Mais, il est inférieur à ceux de $95 \%$ obtenu par Boussim et al. (1993) au Burkina-Faso ; 96 \% enregistré par Soro (2005) au Nord de la Côte d'Ivoire et $87 \%$ obtenu par Ahamidé et al. (2017) dans le Nord Bénin. Par contre, il supérieur à ceux de 60 et 53\% rapportés respectivement par Houénon (2012) et Ahamidé et al. (2015) dans les plantations d'agrumes et celles de Cola nitida au Sud du Bénin. Ce taux est également supérieur à celui de 52,63\% enregistré sur le safourtier (Dacryodes edulis) par Massako et al. (2013) au Cameroun. Ces différences de prévalence des Loranthaceae entre ces études, pourraient être liées d'une part à la densité 
des oiseaux (Pogoniulus chrysonocus) disséminateurs de grains de Tapinanthus, mais aussi à la densité de Colomba guinea, régulatrice du parasitisme des Tapinanthus suivant les zones étudiées. D'autre part, ces prévalences seraient influencées par la perception qu'ont les populations locales sur ces espèces de plantes parasites.

Les résultats ont montré que le Dbh et l'altitude ont un effet significatif sur l'incidence des pieds de karité par les Loranthaceae. Ces résultats corroborent ceux de Houéhanou et al. (2011) et de Dibong et al. (2008), qui ont révélé respectivement des effets significatifs entre le Dbh et le taux d'infestation puis entre l'altitude et le taux d'infestation. En effet, d'après Médah (2001), le regroupement des individus par classe de diamètre révèle que l'âge des sujets serait une fonction importante du parasitisme. Plus le sujet acquiert de l'âge, plus il devient sensible aux plantes parasites. Cela nous fait penser à une éventuelle diminution de la résistance physiologique des espèces au cours de leur vieillesse. De plus, comme dans cette étude, Ahamidé et al. (2015 ; 2017), ont montré que la densité parasitaire augmente avec le Dbh. Ainsi, pour ces auteurs, ce résultat se justifierait par le fait que les arbres les plus gros, donc vieux constituent les refuges pour les oiseaux dans leur quête de sécurité. Or, ces oiseaux sont des agents de transport et de dissémination des graines de plantes parasites. Egalement, le séjour des graines dans le tractus digestif de ces oiseaux facilite la levée de leur dormance.

\section{Conclusion}

Cette étude a montré que Lasiodiplodia theobromae, Colletotrichum gloeosporioides, Pestalotiopsis sp, Xanthomonas sp sont les agents pathogènes des maladies identifiés affectant le karité. $T$. dodoneifolius et $T$. globiferus sont les plantes parasites plus répandus sur le karité avec des incidences moyennes respectives de $68,67 \%$ et de $66,58 \%$ et des densités moyennes par pied respectives de 5,64 touffes et de 2,52 touffes. D'autres plantes épiphytes comme Calyptrochilum christyanum, Ficus thonningii, Alafia barteri sont observées. Une meilleure stratégie de lutte contre ces agents pathogènes et plantes vasculaires passe par une bonne maîtrise des pratiques culturales de gestion au sein des champs de karité.

\section{Remerciements}

Les auteurs remercient l'Association Karité Bénin pour la mise à disposition d'informations sur le karité, Mr Kocou justo pour la conception de la carte et le Dr Houehanou Thierry pour sa contribution scientifique à ce travail. 


\section{References:}

1. Agbahungba, G., \& Depommier, D. (1989). Aspects du parc à karité néré (Vitellaria paradoxa Gaertn.f. Parkia biglobosa) du Borgou (Bénin). Bois et Forêts des Tropiques, 222: 41-54.

2. Ahamidé, D. Y. I., Tossou, M. G., Adomou, A. C., Houénon, G. J., Yédomonhan, H., \& Akoègninou, A. (2015). Diversité, impacts et usages des Loranthaceae parasites de Cola nitida (Vent.) Schott. \&Endl. Au Sud-Bénin. Int. J. Biol. Chem. Sci., 9 (6): 2859-2870.

3. Ahamidé, I. D. Y., Tossou, M. G., Yédomonhan, H., Adomou, A. C., Houénon, J., \& Akoègninou, A. (2017). Diversité des Loranthaceae et leur impact sur Vitellaria paradoxa C.F.Gaertn.: un fruitier à grande valeur socio-économique au Nord-Bénin. European Scientific Journal 13 (24):217-230.

4. Ahouansou, R. H., Sanya, E. A., Bagan, G. C., \& Foudjet, A. E. (2008). Etude de quelques caractéristiques physiques des noix et amandes de karité produites au Bénin. Science et technique, Sciences appliquées et Technologies, 2 (1-2):1-38.

5. Akouehou, G. S., Assogba, D. O., Houndonougbo, A., \& Sinsin, A. B. (2013). Diversité floristique, sécurisation foncière et gestion des systèmes agroforestiers à palmier à huile (Elaeis guineensis) en zones périurbaines et rurales du Département de l'Atlantique au Sud du Bénin. Int. J. Biol. Chem. Sci., 7(3):1180-1189.

6. Akoègninou, A, van der Burg, W. J., \& van der Maesen, L. J. G. (2006). Flore Analytique $d u$ Bénin. Brackhuys Publishers:Wageningen, 1034p.

7. Akrofi, A.Y., \& Amoah, F. M. (2009). Pestalotia spp. Causes leaf spot of Vitellaria paradoxa in Ghana. Cocoa Research Instutute of Ghana, P.O. Box 8, Akim Tafo, Ghana. African Journal of Agricultural Research, 4 (4): 330-333.

8. Amon, A. D. E., Soro, D., N'guessan, K., \& Traoré, D. (2010). Les Loranthaceae: plantes vasculaires parasites des arbres et arbustes, au Sud-est de la Côte d'Ivoire. Journal of Applied Biosciences, 25: 1565 $-1572$.

9. Arbonier, M. (2000). Arbres, arbustes et lianes des zones sèches d'Afrique de l'ouest. CIRAD, MNHN, UICN 2002.

10. Azontonde, A. H. (1991). Propriétés physiques et hydrauliques des sols au Bénin. Soil Water Balance in the Sudano Sahelian Zone. Proceedings of the Niamey Workshop, February 1991. IAHS Publ. no. 199.

11. Azo'o, J. R. N., Tchatat, M., Mony, R., \& Dibong S. D. (2013). Parasitisme et ethnobotanique des Loranthaceae à Lokomo (EstCameroun). Journal of Animal \& Plant Sciences 19 (2): 2923-2932. 
Bayala, J., Ouédraogo, S. J., Ong, C. K. (2009). Early growth performance and water use of planted West African provenances of Vitellaria paradoxa C. F. Gaertn (karité) in Gansé, Burkina Faso. Agroforestry Systems 75:7-127.

12. Bayala, J., Teklehaimanot, Z., \& Ouedraogo, S. J. (2002). Millet production under pruned tree crowns in a parkland system in Burkina Faso. Agroforestry Systems, 54:203-214.

13. Bolker, B. M., Brooks, M. E., Clark, C. J., Geange, S.W., Poulsen, J. R., Stevens, M. H. H., \& White, J.-S. S. (2009). Generalized linear mixed models: a practical guide for ecology and evolution. Trends in Ecology and Evolution, 24 : 127-135.

14. Boussim, I. J. (2002). Les phanérogames parasites du Burkina Faso : inventaire, taxonomie, écologie et quelques aspects de leur biologie. Cas particulier des Loranthaceae parasites du karité. Thèse de doctorat d'état, Université de Ouagadougou, 285 p.

15. Boussim, I. J., Guinko, S., \& Salle, G. (1993). Tapinanthus parasite du karité au Burkina Faso. Bois et Forêts des Tropiques 238: 45-65.

16. Brooks, M. E., Kristensen, K., van Benthem, K. J., Magnusson, A., Berg, C. W., Nielsen, A., \& Bolker, B. M. (2017). glmmTMB Balances Speed and Flexibility Among Packages for Zero-inflated Generalized Linear Mixed Modeling. The R Journal, 9(2) : 378-400.

17. Campbell, C. (2002). Saskatoon Company could revolutionize chocolate industry, The Canadian Connection. IDRC $9 \mathrm{p}$.

18. Codjia, T. J., Assogbadjo, E. A., \& Ekué, M. R. M. (2003). Diversité et valorisation au niveau local des ressources végétales forestières alimentaires du Bénin. Cahiers Agricultures, 12(5): 321-331.

19. Delhove, G., Rey, J-Y., Vannière, H., Pruvost, O. (2013). Bactériose du manguier 1 : bactériose du manguier provoquée par Xanthomonas citri pv. mangiferaeindicae. Bruxelles: Comité de Liaison Europe Afrique Caraïbes pacifique (COLEACP), 14p.

20. Dianda, Z. O., Wonni, P., Zombré, G., Traoré, O., Sérémé, D., Ouédraogo, I., Ouédraogo, S.L., \& Sankara, P. (2018). Prévalence du dessèchement du manguier et évaluation des fréquences des champignons associés à la maladie au Burkina Faso. Journal of Applied Biosciences, 126: 12686-12699.

21. Dibong, D. S., Din, N., Priso, R. J., Taffouo, V. D., Fankem, H., Amougou, A. (2008). Parasitism of host trees by the Loranthaceae in the region of Douala (Cameroon). African Journal of Environmental Science and Technology 11: 371-378.

22. Esiegbuya, D. O., Osagie, J. I., Okungbowa, F. I., Ekhorutomwen, E. O. (2014). Fungi Associated with the Postharvest Fungi Deterioration 
of Shea Nuts and Kernels. International Journal of Agriculture and Forestry, 4 (5): $373-379$.

23. Eyog Matig, O., Gaoué, O. G., Dossou, B. (2002). Réseau «Espèces Ligneuses Alimentaires ». Compte rendu de la première réunion du Réseau tenue 11-13 décembre 2000 au CNSF Ouagadougou, Burkina Faso. Institut International des Ressources Phytogénétiques. 241p. ISBN 92-9043-552-6.

24. Gnanglè, P. C, Egah, J., Baco, M. N., Gbèmavo, C. D. S. J., Kakaï, G. R., \& Sokpon, N. (2012). Perceptions locales du changement climatique et mesures d'adaptation dans la gestion des parcs à karité au Nord-Bénin. Int. J. Biol. Chem. Sci., 6 (1): 136-149.

25. Gnanglè, C. P. Yabi, A. J., Glèlè Kakaï, J. L. R., \& Sokpon, N. (2009). Changements climatiques: Perceptions et stratégies d'adaptations des paysans face à la gestion des parcs à karité au Centre Bénin. www.sifee.org/Actes/actes_niamey /1_GNANGLE_comm.pdf; 1-18.

26. Hill, M. O. (1973). Diversity and Evenness: A Unifying Notation and Its Consequences. Ecology, 54: 427-432.

27. Houéhanou, T. D., Kindomihou, \& V., Sinsin, B. (2011). Effectiveness of Conservation areas in protecting Shea trees against Hemiparasitic plants (Loranthaceae) in Benin, West Africa. Pl. Ecol. Evol., 144 (3): 267-274.

28. Houénon, G. J. (2012). Les Loranthaceae des Zones Guinéenne et Soudano-Guinéenne au Bénin et leur Impact sur les plantations Agrumicoles. Thèse de Doctorat, Université d'Abomey-Calavi, Abomey-Calavi, $133 \mathrm{p}$.

29. Jiofack, T., Dondjang, J. P., \& Nkongmeneck, B. A. (2007). Les Loranthaceae du groupement Bafou: identification, distribution, biologie et contrôle. www. tela-botanica.org/Les Loranthaceae du groupement bafou/doc.

30. Kapseu, C., Dirand, M., Jiokap, N.Y., Parmentier, M. \& Dellacherie, J., (2001). Absorption de l'eau : Influence de condition de traitement des amandes de karité et de la pulpe de l'aiélé. Communication présentée à la 9e conférence du comité camerounais de Biosciences. Limbé.

31. Kindt, R., Kalinganire, A., Larwanou, M., Belem, M., Dakouo, J. M., Bayala, J. \& Kaire, M. (2008). Species accumulation within land use and tree diameter categories in Burkina Faso, Mali, Niger and Senegal. Biodiversity and Conservation, 17: 1883-1905

32. Kpadonou, R. A. B., Adégbola, P. Y., \& Tovignan, S. D. (2012). Local knowledge and adaptation to climate change in Ouémé valley, Benin. African Crop Science Journal, 20 (2): 181-192. 
33. Lafleur, M. (2008). Recherche et documentation des meilleurs pratiques pour la gestion durable des parcs à Karité en Afrique de l'Ouest. Québec, Canada. 110 p.

34. Larrue, S. (2005). Contribution au débat sur l'absence relative de karité au Sénégal oriental : fondements naturels, raisons sociales ? », Les Cahiers d'Outre-Mer, 229 [En ligne], mis en ligne le 13 février 2008. URL : http://com.revues.org/index477.html. Consulté le 11 août 2010.

35. Massako, F., Tchatat, M., Mony, R., Ladoh, Y. C. F., \& Dibong, S. D. (2013). Parasitisme de Dacryodes edulis par le genre Tapinanthus (Loranthaceae) et répartition de la Myrmécofaune associée à Logbessou Plateau (Douala, Cameroun). Journal of Applied Biosciences, 68: 5336- 5348.

36. Médah, N. (2001). Contribution à la lutte contre les plantes parasites des ligneux à la Station de Recherches Environnementales et Agricoles de Saria. Mémoire de fin d'études en vue de l'obtention du Diplôme d'Ingénieur du Développement Rural, Option: Eaux et Forêts. Université Polytechnique de Bobo-Dioulasso (UPB). Burkina-Faso. $89 \mathrm{p}$.

37. Ministère de l'Agriculture de l'Elevage et de la Pêche (MAEP). (2008). Atelier d'élaboration des stratégies des chaînes de valeur du karité au Bénin. Centre Guy Riobé de Parakou.

38. Odebiyi, J. A., Bada, S. O., Omoloye, A. A., Awodoyin, R. O., \& Oni, P. I. (2004). Vertebate and insect pests and hemi-parasitic plants of Parkia biglobosa and Vitellaria paradoxa in Nigeria. Agroforestry Systems 60 : 51-59.

39. Pruvost, O., Boyer, O., Vital, K., Verniere, C. \& Gagnevin, L. de Bruno Austin L. \& Ray, J. Y. (2011). First report in Ghana of Xanthomonas citri pv. mangiferaeindicae causing mango bacterial canker on Mangifera indica. American Phytopathological Society, 95 (6): 774.

40. R Core Team (2018). A Language and Environment for Statistical Computing. $R$ Foundation for Statistical Computing, Vienna. https://www.R-project.org

41. Rehman, A., Din Umar, U., Naqvi, S. A. H., Latif, M. R., Khan, S. A., Malik, M. T. \& Freed, S. (2015). Emerging resistance against different fungicides in Lasiodiplodia theobromae as the cause of mango dieback in Pakistan. Arch. Biology Sciences. 67 (1): 241-249.

42. Sakalidis, M. L., Hardy, G. E., \& Burgess, T. I. (2011). Pathogenic Botryosphaeriaceae associated with Mangifera indica in the Kimberley region Of Western Australia. In: European Journal Plant Pathology 130: 379-391. 
43. Samaké, O., Dakouo, J. M., Kalinganire, A., Bayala, J., \& Koné, B. (2011). Techniques de déparasitage et gestion du karité. ICRAF Technical Manual No. 15. Nairobi: World Agroforestry Centre.

44. Sijaona, M. E. R., Clewer, A., Maddison, A., \& Mansfield, J. W. (2001). Comparative analysis of powdery mildew development on leaves, seedlings and flower panicles of different genotypes of cashew. Plant Pathology 50 (2): 234-243.

45. Sodjinou, E. (2006). Institutions locales traditionnelles et modernes de gestion des ressources naturelles, des situations conflictuelles et divers conflits autour de ces ressources au Bénin. PAPA-INRABIFS, Bénin, $1-128$.

46. Soro, K. (2005). Les Loranthaceae des légumineuses arborées introduites dans la zone forestière d'Oumé : espèces rencontrées, spectres d'hôtes et taux d'infestation. Mémoire de DEA, Université de Cocody, Abidjan, $51 \mathrm{p}$.

47. Tédihou, E., Kpémoua, K., \& Tounou, A. (2017). Dépérissement des manguiers et citrus dans la région centrale du Togo et méthodes de lutte par des fongicides. Journal of Applied Biosciences 119: 1182911838.

48. Watanabe, T. (1994). Pictorial of Atlas of Soil and Seed Fungi: Morphologies of cultured fungi and key to species. CRC Press, Inc. Lewis Publishers. 411p.

49. Zinsou, V. A., Ferdinan, A., Tchémadon, C. G., Afouda, L. A. C., \& Gagnon, F. (2017). Inventaire des principales maladies du manguier dans les savanes soudanienne et nord guinéenne du Bénin. Ann. UP, Série Sci. Nat. Agron. 7 (1): 25-31. 